\title{
High-sensitivity Hydrogen Leakage Sensor for New-energy Vehicles
}

\author{
Jing $\mathrm{Ji}^{1}{ }^{*}$ Meng Zhao, ${ }^{1}$ and Toshitsugu Ueda ${ }^{2}$ \\ ${ }^{1}$ School of Electro-Mechanical Engineering, Xidian University, No. 2 South Taibai Road, Xi'an 710071, P. R. China \\ ${ }^{2}$ IPS Research Center, Waseda University, 2-2 Hibikino, Wakamatsu-ku, Kitakyushu, Fukuoka 808-0135, Japan
}

(Received July 27, 2020; accepted January 15, 2021)

Keywords: hydrogen sensor, quartz resonator, optimal design, energy trapping, new-energy vehicles

In this paper, we report a high-sensitivity hydrogen leakage sensor for new-energy vehicles based on our originally designed AT-cut quartz resonator. Firstly, as optimization work, we established a finite element model and carried out an eigenfrequency analysis to find the optimal dimensions of the sensor. Secondly, as verification work, the sensor was fabricated using our newly developed etching process and evaluated. The simulated frequency was basically in agreement with the measured frequency, and good energy-trapping performance and decoupling characteristics were realized, which confirm the validity of our optimal design. Finally, the sensitivity of the sensor was examined experimentally, and good sensitivity was achieved.

\section{Introduction}

As an abundant and clean energy resource, hydrogen gas has a low environmental impact and is attracting attention as a potential resource to replace gasoline and diesel fuels. In recent decades, promoted by governments and major automobile groups, hydrogen fuel cells have been widely applied in new-energy vehicles. However, hydrogen is a flammable and explosive gas. The explosive limit of hydrogen in air ranges from about 18 to $60 \%$, and the flammable limit is from 4 to $75 \% .{ }^{(1)}$ Various hydrogen leakage sensors ${ }^{(2,3)}$ have been developed, which include contact combustion type, semiconductor type, thermoelectric type, and ball surface acoustic wave (SAW) type (ultrasonic type). Hydrogen safety is one of the most important safety indicators of new-energy vehicles, and there is still increasing demand for hydrogen leakage sensors with a fast response, high sensitivity, and new detection methods. Further effort is required in basic research, especially on new materials and sensor principles, to fully meet the requirements of emerging technical applications.

In this paper, we present a new originally designed hydrogen leakage sensor based on an ATcut quartz resonator. The AT-cut quartz crystal was selected because of its good temperature coefficient of frequency (TCF) and the applicability of a newly developed etching technology. ${ }^{(4)}$ The fundamental thickness shear (TS) mode was selected as the main vibration mode, which is a stable mode and less influenced by the surrounding gas at atmospheric pressure.

*Corresponding author: e-mail: jingji@xidian.edu.cn https://doi.org/10.18494/SAM.2021.2993 
Our newly designed quartz crystal hydrogen sensor has a rectangular AT-cut quartz resonator (see Fig. 1), which has two excitation electrodes of platinum (Pt) and gold ( $\mathrm{Au}$ ) on the upper surface of the quartz plate and one common electrode on the lower surface. Chromium $(\mathrm{Cr})$ is used as an adhesive layer. The upper $\mathrm{Pt}$ electrode has a $\mathrm{Cr} / \mathrm{Au} / \mathrm{Pt}$ three-layer structure and the upper $\mathrm{Au}$ electrode has a $\mathrm{Cr} / \mathrm{Au}$ two-layer structure. The lower Pt electrode has a $\mathrm{Pt} / \mathrm{Cr}$ two-layer structure. The thickness of the quartz plate is determined by the AT-cut quartz wafer, which is $100 \mu \mathrm{m}$. The thickness of the $\mathrm{Cr}$ is fixed at $0.05 \mu \mathrm{m}$. Au is used as a conductive layer with thickness fixed at $0.1 \mu \mathrm{m}$. The thickness of the Pt film $T$ was optimized in this work. In our previous work, ${ }^{(5,6)}$ we discussed the influence of the dimensions of the two-layer electrode on the performance of quartz resonators. This paper reports the first optimization of the design for a quartz resonator with a three-layer electrode.

For the quartz plate resonator, the main vibration mode is the fundamental TS mode. The theoretical frequency of the TS vibration ${ }^{(7)}$ is given as

$$
\begin{gathered}
f=f_{0}+\Delta f, \\
f_{0}=\frac{\sqrt{G / \rho}}{2 b}, \\
\Delta f=\frac{-2 f_{0}^{2} \Delta M}{A \sqrt{\rho G}},
\end{gathered}
$$

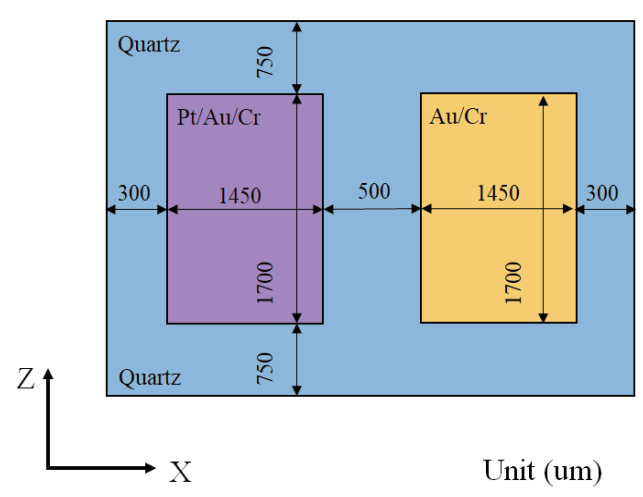

(a)
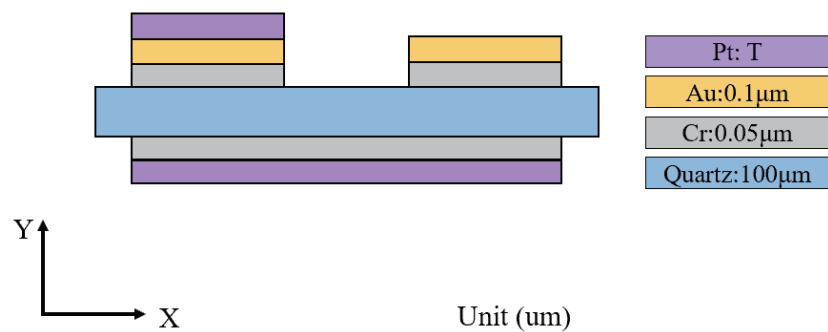

Unit (um)

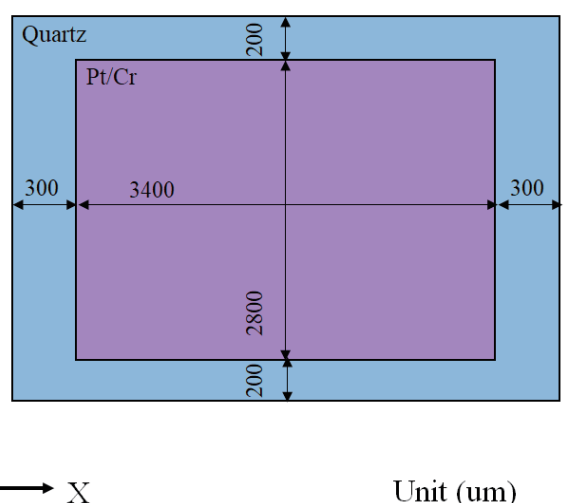

(b)

(c)

Fig. 1. (Color online) Profile of the hydrogen sensor. (a) Top view. (b) Bottom view. (c) Section view. 
where $f_{0}$ is the fundamental TS mode frequency without the electrode and $\Delta f$ is the frequency shift due to the mass loading effect of the electrode, given by the well-known Sauerbrey equation. ${ }^{(8)}$ $G$ is the shear modulus of the AT-cut quartz, $\rho$ is its density, $b$ is the quartz plate thickness, $\Delta M$ is the electrode mass, and $A$ is the electrode surface area.

An electrode with different materials and dimensions causes a different frequency shift. Therefore, the two upper $\mathrm{Pt}$ and $\mathrm{Au}$ electrodes make the quartz resonator vibrate at two separate frequencies. The lower Pt/Cr electrode is used as a heater, which can self-heat up to $100{ }^{\circ} \mathrm{C}$, where $\mathrm{Pt}$ is used as a catalyst for the catalytic reaction between hydrogen and oxygen gases given by the chemical equation

$$
2 \mathrm{H}_{2}+\mathrm{O}_{2}=2 \mathrm{H}_{2} \mathrm{O}+\text { Heat generated. }
$$

When the temperature rises due to the catalytic reaction at the Pt electrode surface in the hydrogen gas environment, the vibration frequencies of the two electrodes change, which makes it possible to measure the hydrogen gas concentration.

\section{Optimal Design of Hydrogen Sensor}

For the quartz plate resonator, the fundamental TS vibration mode is generated in the $x-y$ plane. Therefore, the energy-trapping effect and decoupling characteristic of the resonator are mainly related to unwanted vibrations including inharmonic overtone (IO) and thicknessflexural (TF) vibrations, which are also generated in the $x-y$ plane. More unwanted vibrations lead to greater energy loss and stronger couplings. Theoretically, if the dimension in the $x$-direction of the resonator is prespecified, the frequency of spurious vibrations is only dependent on the dimension of the resonator in the $y$-direction. ${ }^{(9)}$ Therefore, in this work, the thickness of the Pt film was the important parameter that needed to be optimized.

In the modeling process, to ensure calculation accuracy and save calculation time, linear quadrilateral type elements were used to establish a two-dimensional finite element model using COMSOL MULTIPHYSICS ${ }^{\mathrm{TM}}$. The values used for the materials were from IEEE Standard on Piezoelectricity, ${ }^{(10)}$ the materials library of COMSOL MULTIPHYSICSTM, and Yang et al.'s report. ${ }^{(11)}$

The elastic constant of AT-cut quartz is expressed in matrix form as

$$
C^{E}{ }_{i j}=\left[\begin{array}{cccccc}
86.74 & -8.25 & 27.15 & -3.66 & 0 & 0 \\
-8.25 & 129.77 & -7.42 & 5.7 & 0 & 0 \\
27.15 & -7.42 & 102.83 & 9.92 & 0 & 0 \\
-3.66 & 5.7 & 9.92 & 38.61 & 0 & 0 \\
0 & 0 & 0 & 0 & 68.81 & 2.53 \\
0 & 0 & 0 & 0 & 2.53 & 29.01
\end{array}\right] \times 10^{9}(\mathrm{~Pa})
$$

The piezoelectric constant and dielectric constant matrices for AT-cut quartz are, respectively, 


$$
\begin{gathered}
e=\left[\begin{array}{cccccc}
-0.171 & 0.152 & 0.0187 & -0.067 & 0 & 0 \\
0 & 0 & 0 & 0 & -0.108 & 0.095 \\
0 & 0 & 0 & 0 & 2.53 & 29.01
\end{array}\right]\left(\mathrm{C} / \mathrm{m}^{2}\right), \\
\varepsilon=\left[\begin{array}{ccc}
39.21 & 0 & 0 \\
0 & 39.82 & 0.86 \\
0 & 0.86 & 40.42
\end{array}\right] \times 10^{-12}(\mathrm{~F} / \mathrm{m}) .
\end{gathered}
$$

The parameters of the $\mathrm{Pt}, \mathrm{Au}$, and $\mathrm{Cr}$ electrodes are shown in Table 1. Table 2 shows the meshing conditions. The meshing mode of the hydrogen sensor is shown in Fig. 2. There are two TS vibration modes for the upper Pt and Au electrodes, as shown in Fig. 3.

Firstly, we carried out eigenfrequency analysis to calculate the two TS vibration modes for different thicknesses of the Pt film $T$, where $T$ was changed from 0.1 to $0.3 \mu \mathrm{m}$ with $0.05 \mu \mathrm{m}$ steps. Figure 4 shows that with increasing $T$, the frequency of the TS mode for the Au electrode

Table 1

Parameters of electrodes.

\begin{tabular}{lccc}
\hline Parameter & $\mathrm{Cr}$ & $\mathrm{Au}$ & $\mathrm{Pt}$ \\
\hline Density $\left(\mathrm{kg} / \mathrm{cm}^{3}\right)$ & $7.19 \times 10^{3}$ & $19.30 \times 10^{3}$ & $21.45 \times 10^{3}$ \\
\hline Young's modulus $(\mathrm{GPa})$ & 279 & 79 & 169 \\
\hline Poisson ratio & 0.21 & 0.42 & 0.38 \\
\hline
\end{tabular}

Table 2

Meshing conditions.

\begin{tabular}{lcccc}
\hline \multirow{2}{*}{ Mesh number } & \multirow{2}{*}{ Quartz plate } & \multicolumn{4}{c}{ Upper electrode Upper electrode Lower electrode } \\
& & $\mathrm{Pt} / \mathrm{Au} / \mathrm{Cr}$ & $\mathrm{Au} / \mathrm{Cr}$ & $\mathrm{Pt} / \mathrm{Cr}$ \\
\hline$X$-direction & 4000 & 1450 & 1450 & 3400 \\
$Y$-direction & 100 & 1 & 1 & 1 \\
\hline
\end{tabular}

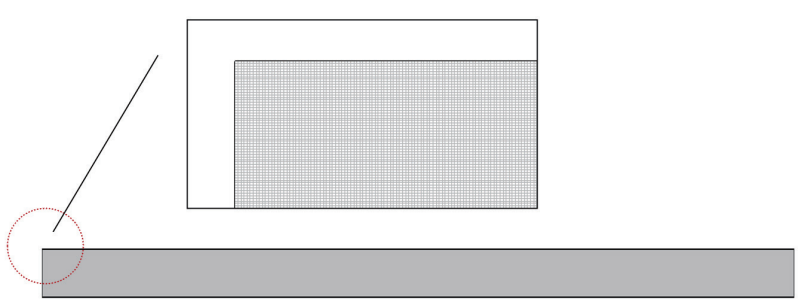

Fig. 2. (Color online) Finite element modeling of sensor.

(a)

(b)

Fig. 3. (Color online) TS modes for (a) Pt electrode and (b) Au electrode. 


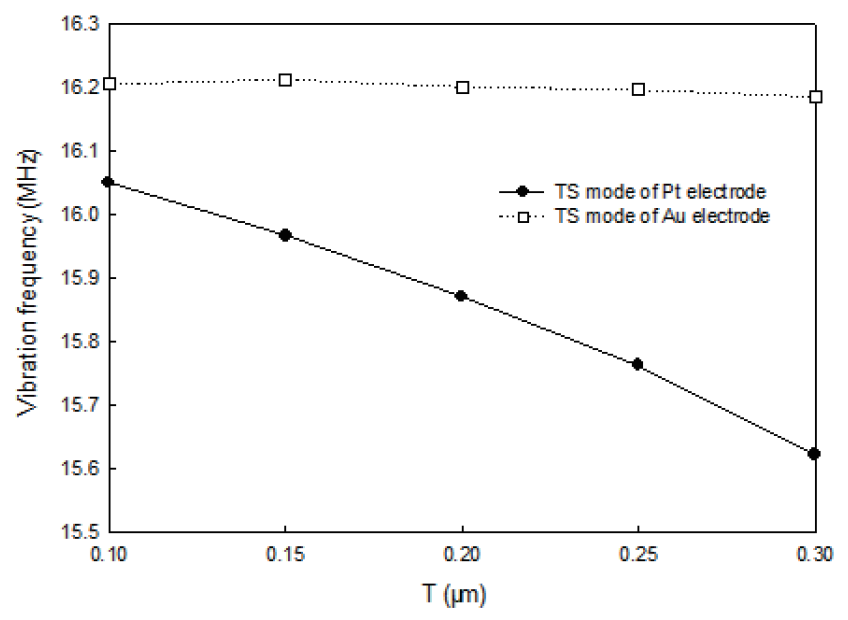

Fig. 4. Frequencies of TS modes of Pt and Au electrodes for different $T$ values.

decreased slowly but the frequency of the TS mode for the Pt electrode decreased rapidly, and the difference between the two frequencies also increased. In particular, when $T$ was larger than $0.2 \mu \mathrm{m}$, the difference between the two frequencies was larger than $0.4 \mathrm{MHz}$, which is enough to distinguish the two vibration modes.

Secondly, we examined the performance of energy trapping and the decoupling characteristic for the Pt electrode mode. Figure 5 shows the calculated $X$-displacement line (the line was parallel to the $X$-axis with its $Y$ coordinate equal to $100 \mu \mathrm{m}$ ) distribution in the TS mode for the Pt electrode for different $T$ values. The red line was obtained by linear regression analysis to recover the pure fundamental TS vibration. It was shown that some relatively small fluctuations existed along the distribution line, which were caused by coupling between TS and TF vibrations. When $T$ increased to $0.25 \mu \mathrm{m}$, the fluctuations became larger, and stronger coupling occurred. For other $T$ values, the fluctuations were vanishingly small and the coupling strength was acceptable. On the other hand, according to Sauerbrey's theory, the thicker the electrode, the more energy is trapped in the electrode region. Therefore, considering the above, 0.2 and $0.3 \mu \mathrm{m}$ were selected as optimal values of the Pt electrode thickness.

Thirdly, we examined the performance of energy trapping and the decoupling characteristic for the $\mathrm{Au}$ electrode mode. Figure 6 shows a calculated $X$-displacement line distribution in the TS mode for the Au electrode. It was shown that the energy-trapping performance and decoupling characteristic were poor as there were two small peaks caused by IO vibration in addition to the large peak caused by the fundamental TS vibration. It is necessary to reduce the IO vibration for the Au electrode mode. We therefore calculated the $X$-displacement line distribution in the TS mode for different $T$ values (Figs. 6-10).

According to Figs. 7 and 10, when $T$ was 0.15 and $0.3 \mu \mathrm{m}$, the spurious coupling caused by the IO mode was relatively large. According to Figs. 6, 8, and 9 when $T$ was $0.1,0.2$, and 0.25 $\mu \mathrm{m}$, respectively, relatively weak coupling was obtained.

To quantitatively describe the strength of this kind of coupling, we defined the IO coupling coefficient as 


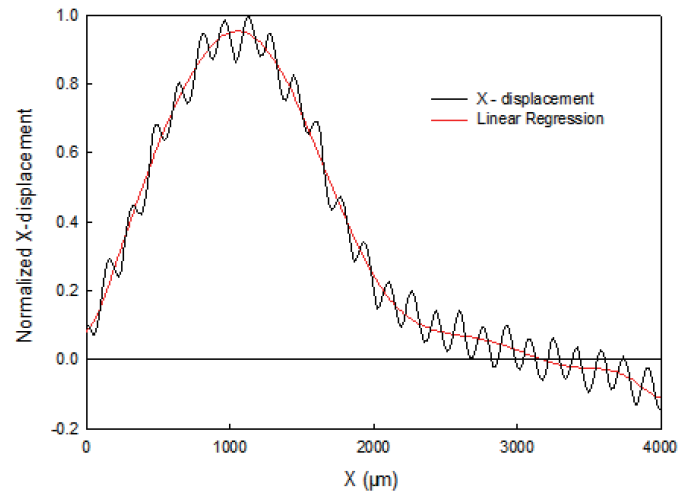

(a)

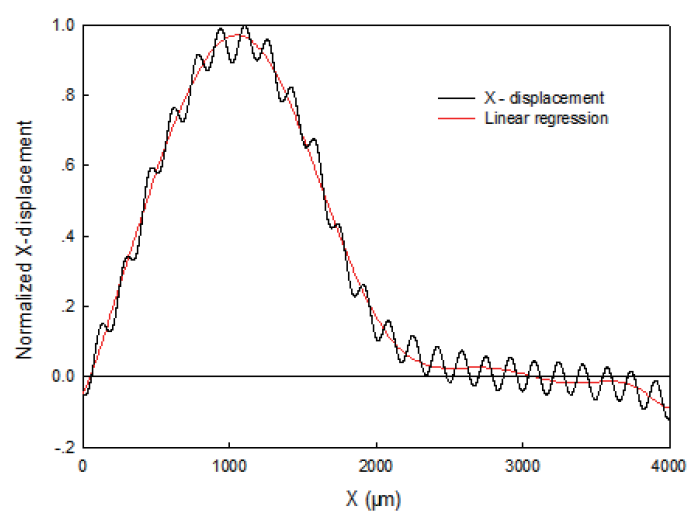

(c)

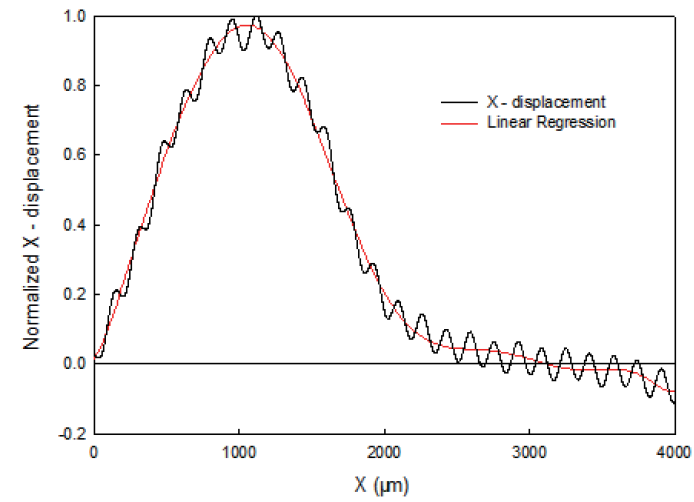

(b)

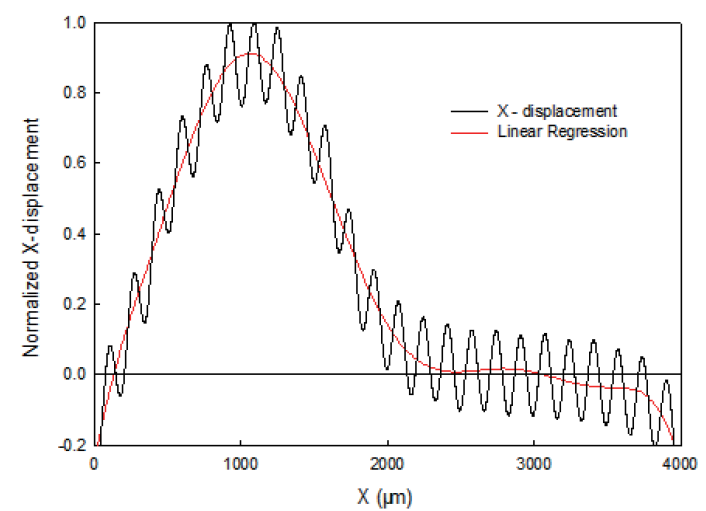

(d)

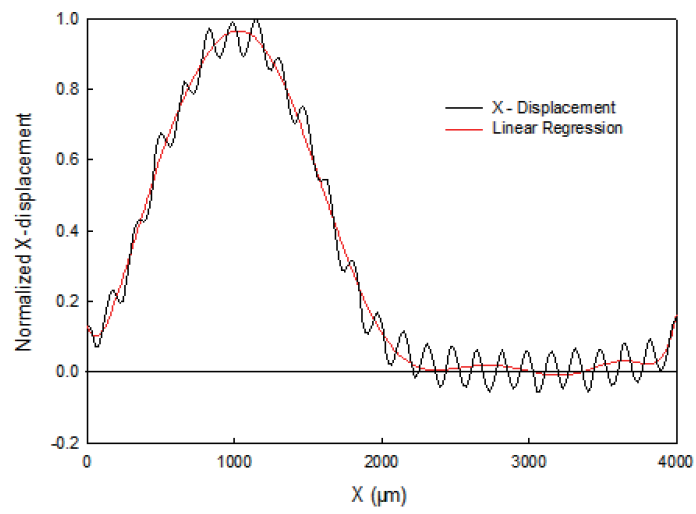

(e)

Fig. 5. (Color online) $X$-displacement line distribution in TS mode of Pt electrode. (a) $T=0.1 \mu \mathrm{m}$, (b) $T=0.15 \mu \mathrm{m}$, (c) $T=0.2 \mu \mathrm{m}$, (d) $T=0.25 \mu \mathrm{m}$, and (e) $T=0.3 \mu \mathrm{m}$.

$$
C=\frac{D_{C}}{D_{T}}
$$

where $D_{C}$ denotes the maximum extra displacement caused by IO vibration and $D_{T}$ denotes the ideal maximum displacement caused by pure fundamental TS vibration. 


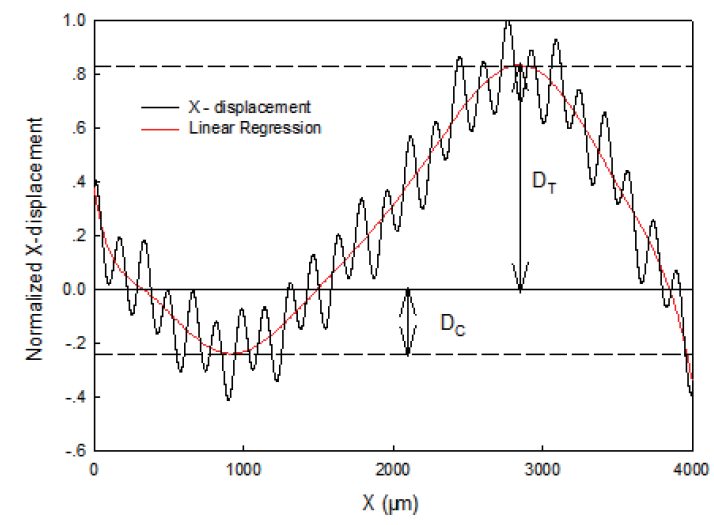

Fig. 6. (Color online) $X$-displacement line distribution in TS mode of Au electrode. $(T=0.1 \mu \mathrm{m})$

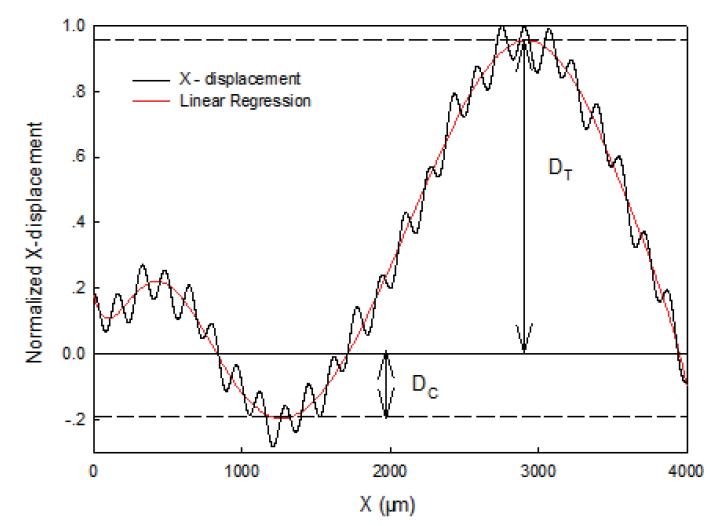

Fig. 8. (Color online) $X$-displacement line distribution in TS mode of Au electrode. $(T=0.2 \mu \mathrm{m})$

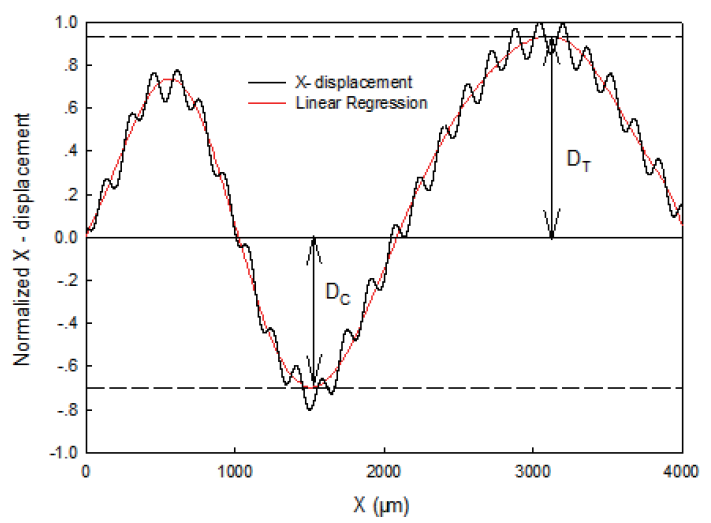

Fig. 7. (Color online) $X$-displacement line distribution in TS mode of Au electrode. $(T=0.15 \mu \mathrm{m})$

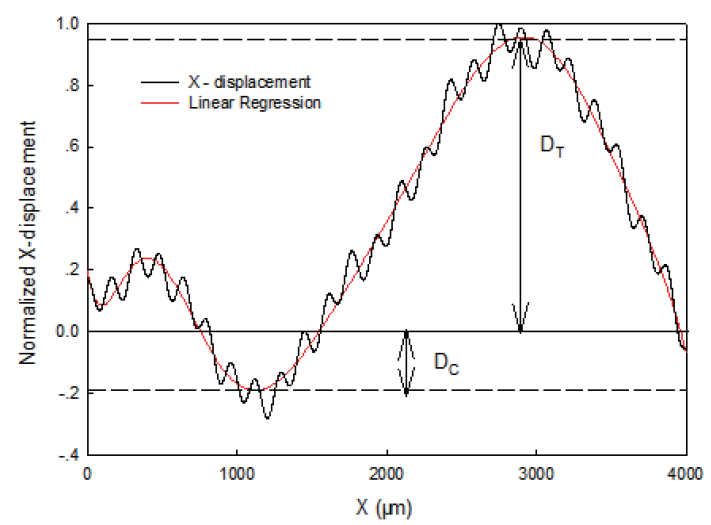

Fig. 9. (Color online) $X$-displacement line distribution in TS mode of Au electrode. $(T=0.25 \mu \mathrm{m})$

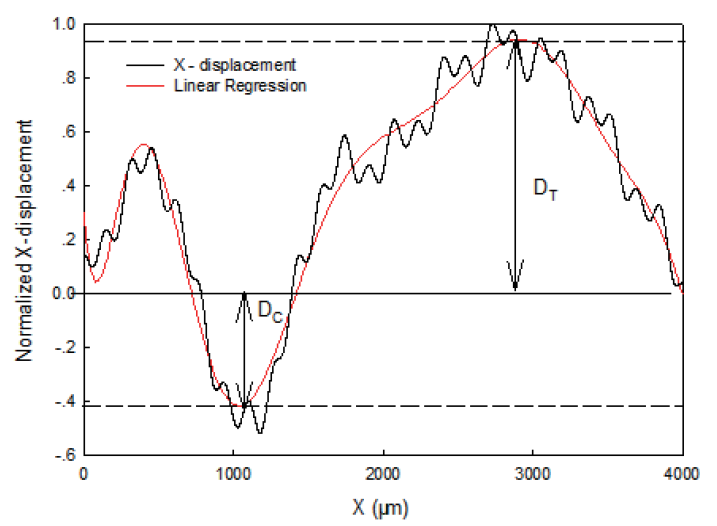

Fig. 10. (Color online) $X$-displacement line distribution in TS mode of Au electrode. $(T=0.3 \mu \mathrm{m})$.

The coupling coefficients were calculated for the sensors with different $T$ values. According to Fig. 11, when $T$ was 0.2 and $0.25 \mu \mathrm{m}$, the coupling coefficient remained stable and small. Finally, after considering the above performance of not only the Pt electrode mode but also the Au electrode mode, $0.2 \mu \mathrm{m}$ was deemed as the optimal value of $T$. 


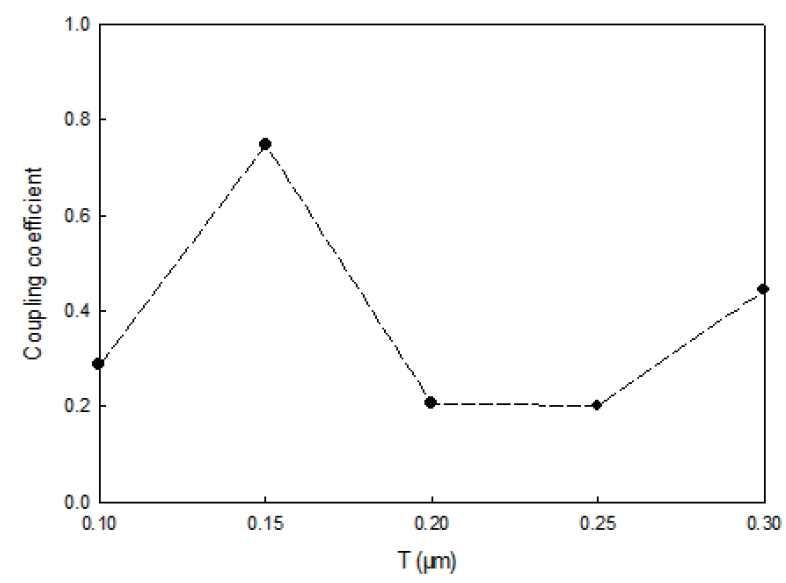

Fig. 11. Coupling coefficient for Au electrode for different $T$ values.

\section{Verification}

We fabricated hydrogen sensors with the optimal Pt film thickness $(0.2 \mu \mathrm{m})$. The manufacturing process had the following main steps: (1) cleaning of the quartz wafer; (2) sputtering of $\mathrm{Cr}$ and $\mathrm{Au}$; (3) photoresist coating and baking; (4) exposure and development; (5) sputtering of Pt; (6) etching of $\mathrm{Au}$; (7) Pt lift-off patterning; (8) etching of quartz. Fabricated and packaged sensors are shown in Fig. 12.

We used an impedance analyzer (Agilent 4294A, $40 \mathrm{~Hz}$ to $110 \mathrm{MHz}$ ), a transmission/ reflection test set (87512A, DC to $2 \mathrm{GHz}$ ), a high-frequency probe (ESST coaxial probe arm A-C1, DC to $3 \mathrm{GHz}$ ), and a manual prober to obtain the frequency response of a fabricated hydrogen sensor (Fig. 13).

Figure 13 shows that, not only for the Pt electrode mode but also for the Au electrode, the responses of the spurious vibration were relatively weak compared with the response of the TS mode and were far from the TS mode, which means that the energy-trapping performance and decoupling characteristic of the optimized sensor were satisfactory.

The frequencies calculated from the simulation were compared with the measured values of our fabricated sensor. As shown in Table 3, the simulation results basically tally with the measured results, confirming the validity of our design.

The characteristics of the fabricated hydrogen sensor were measured using our designed hydrogen testing equipment. Figure 14 shows the schematic and photographs of experimental devices. A mass flow controller (HORIBASTEC, SEC-E40) was used to adjust the concentrations of three gases: air, hydrogen gas, and nitrogen gas. The sensor was placed in the chamber where the gases were mixed and a catalytic reaction between hydrogen and oxygen occurred. A rotary pump was used to exhaust undesired gases from inside the chamber to outside.

In the experiment, we mixed the hydrogen gas and air. The hydrogen concentration was varied from 0 to $6 \%$ and the temperature of the chamber was set to $100{ }^{\circ} \mathrm{C}$ in order that the 

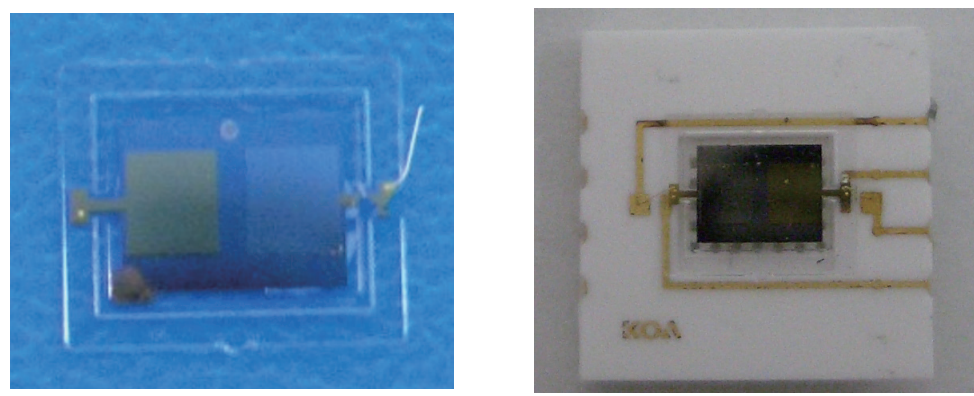

Fig. 12. (Color online) Fabricated and packaged sensors.
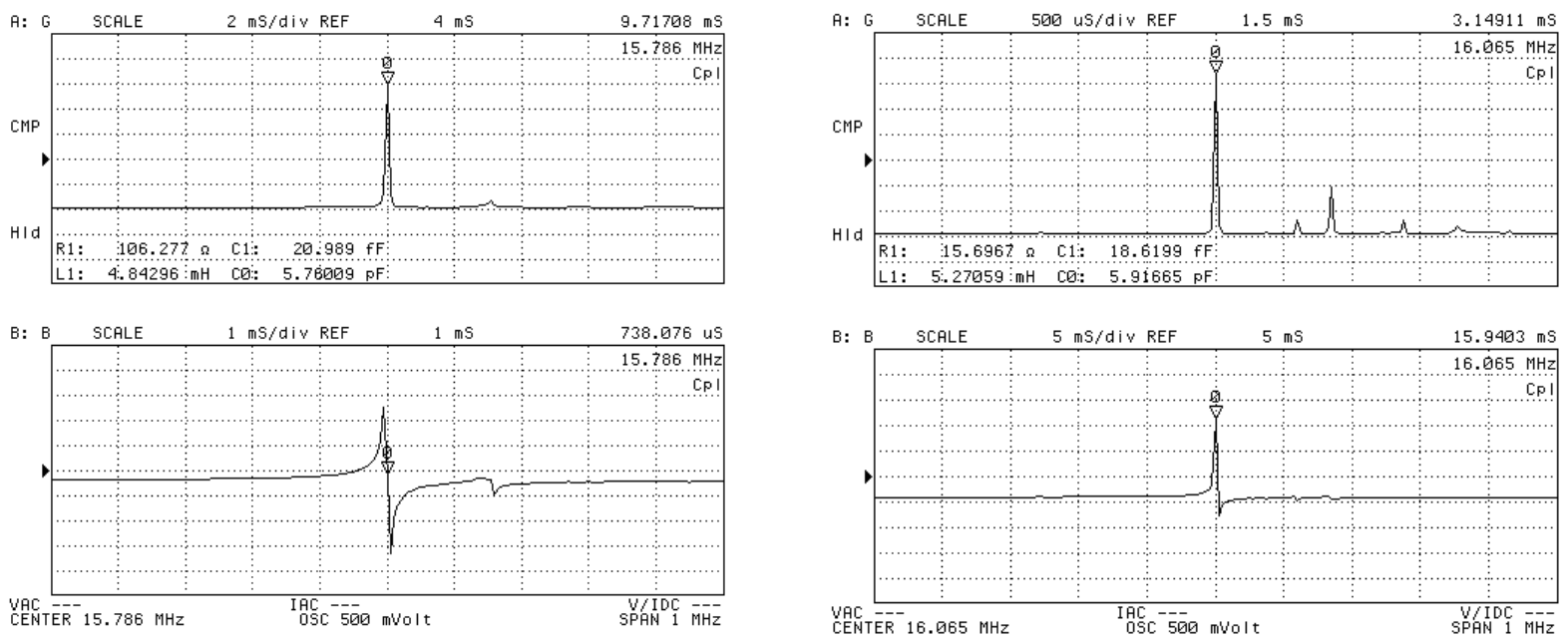

(a)

(b)

Fig. 13. Frequency response of the fabricated hydrogen sensor: (a) Pt electrode mode and (b) Au electrode mode.

Table 3

Comparison of simulated and measured frequencies.

\begin{tabular}{lcc}
\hline & Pt electrode & Au electrode \\
\hline Simulated results (MHz) & 15.87 & 16.20 \\
Measured results (MHz) & 15.786 & 16.065 \\
Error (\%) & 0.53 & 0.84 \\
\hline
\end{tabular}

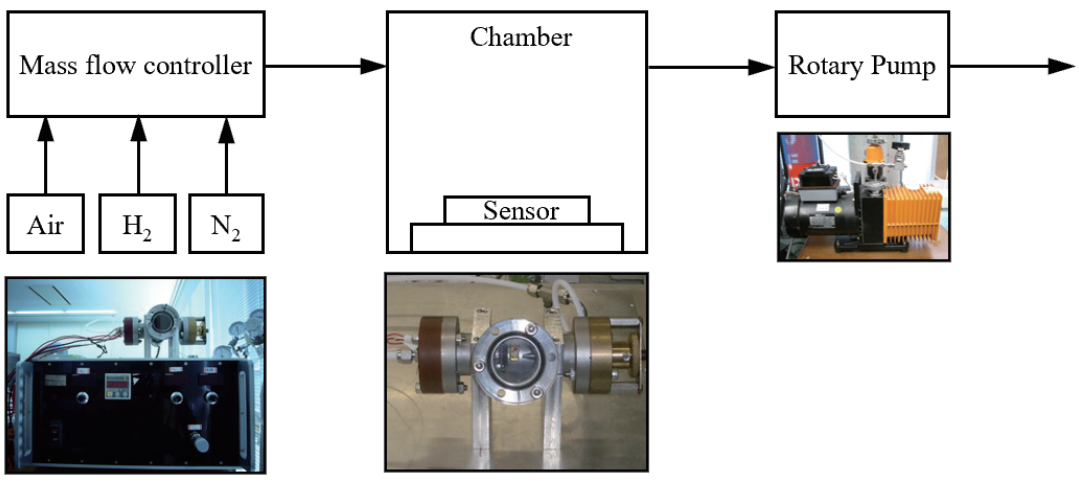

Fig. 14. (Color online) Schematic and photographs of experimental devices. 
catalytic reaction between hydrogen and oxygen in air readily occurred on the Pt electrode surface. The frequency shifts of the Pt and Au electrodes caused by the reaction heat were measured by an impedance analyzer. The sensitivity of the sensor was determined by the relative frequency shift $\Delta f / f$ for each $1 \%$ increase in the hydrogen concentration. We found that the average value was $4 \mathrm{ppm}$. In particular, when the hydrogen concentration increased from 3 to $4 \%$, the relative frequency shift was $7 \mathrm{ppm}$, which shows the high sensitivity of the sensor.

\section{Conclusions}

In this paper, we reported a high-precision hydrogen leakage sensor based on an AT-cut quartz resonator that can be applied to new-energy vehicles. Firstly, as optimization work, we established a finite element model and carried out eigenfrequency analysis to find the optimal dimensions of the sensor. Secondly, as verification work, the sensor was fabricated using our newly developed etching process and evaluated. The simulated frequency was basically in agreement with the measured frequency, and good energy-trapping performance and decoupling characteristics were realized, which confirm the validity of our optimal design. Finally, the sensitivity of the sensor was examined in an experiment, and a good sensitivity of $7 \mathrm{ppm}$ was achieved.

In future work, we will try to build a specific test environment (e.g., a large sealed chamber) to contain a whole vehicle and apply multiple hydrogen sensors to detect the leakage and emission of hydrogen gas around the vehicle. We will also consider how to design our hydrogen sensor using a quartz crystal with a different cut angle (e.g., SR-cut quartz crystal).

\section{References}

1 Y. Hajji, B. Jouini, M. Bouteraa, A. Elcafsi, A. Belghith, and P. Bournot: Int. J. Hydrog. Energy 40 (2015) 9747.

2 K. Tajima, L. F. Houlet, W. Shin, T. Itoh, N. Izu, and I. Matsubara: Jpn. J. Appl. Phys. 45 (2006) 6186.

3 K. Yamanaka, S. Ishikawa, N. Nakaso, and N. Takeda: Proc. 2003 IEEE Ultrasonics Symp. (IEEE, 2003) 299-302.

4 M. Zhao and J. Ji: Sens. Mater. 30 (2018) 1053. https://doi.org/10.18494/SAM.2018.1833

5 J. Ji, H. Oigawa, H. Chen, M. Zhao, and T. Ueda: IEEJ Trans. Sens. Micromach. 132 (2012) 275. https://doi. org/10.1541/ieejsmas.132.275

6 J. Ji, M. Zhao, H. Oigawa, and T. Ueda: Sens. Mater. 30 (2018) 1063. https://doi.org/10.18494/SAM.2018.1832

7 R. Higuchi and Y. Kanno: Jpn. J. Appl. Phys. 45 (2006) 4232. https://doi.org/10.1143/JJAP.45.4232

8 G. Sauerbrey: Zeitschrift für Physik 155 (1959) 206. https://doi.org/10.1007/BF01337937

9 R.D. Mindlin and P.C.Y. Lee: Int. J. Solids Structures 2 (1966) 125. https://doi.org/10.1063/1.361387

10 IEEE Standard on Piezoelectricity, ANSI/IEEE Std 176 (1987). https://doi.org/10.1109/IEEESTD.1988.79638

11 J. Yang, H. Zhou, and W. Zhang: IEEE Trans. Ultrason. Ferroelectr. Freq. Control 52 (2005) 918. https://doi. org/10.1109/TUFFC.2005.1503978 


\section{About the Authors}

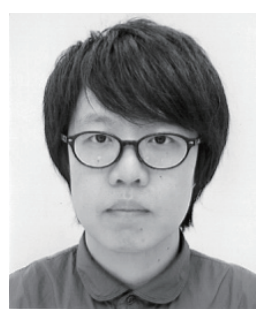

Jing Ji received his B.Sc. degree in microelectronic engineering from Nankai University, China, in 2003, and his M.S. and Ph.D. degrees in the engineering of information, production, and systems from the Graduate School of Information, Production and Systems, Waseda University, Japan, in 2007 and 2013, respectively. From 2013 to 2016, he worked as a research associate and researcher in the Information, Production and Systems Research Center, Waseda University. Since 2016, he has been an associate professor at Xidian University, China. He is a member of the Institute of Electrical and Electronics Engineers (IEEE), the Institute of Electrical Engineers of Japan (IEEJ), and the Japan Society of Applied Physics (JSAP). His research areas include quartz MEMS devices, the finite element method, and sensing technology.

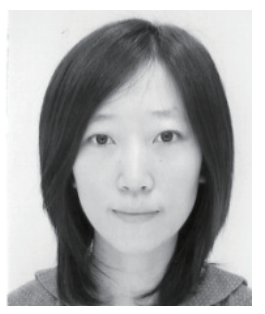

Meng Zhao received her B.Sc. degree in physics from Nankai University, China, in 2003. She received her Ph.D. degree in astrophysics from Graduate University of the Chinese Academy of Sciences in 2009. From 2010 to 2015, she worked as a research associate and researcher in the Information, Production and Systems Research Center, Waseda University, Japan. Since 2016, she has been an associate professor at Xidian University, P.R. China. She is engaged in the research and development of an anisotropic wet etching simulator of quartz crystal.

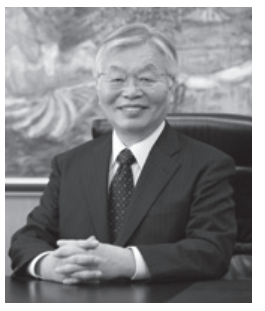

Toshitsugu Ueda received his B.E. and M.E. degrees in electrical engineering from Shinshu University, Nagano, Japan, in 1969 and 1971, respectively. He received his Ph.D. degree from Tokyo Institute of Technology in 1988. Since joining Yokogawa Electric Corporation in 1971, he has been engaged in developing low-noise amplifiers, mechanical resonators, micromachining technologies, and sensors using these technologies to control temperature, pressure, and displacement. He became a professor at the Graduate School of Information, Production and Systems (IPS), Waseda University, in 2003 and Dean of IPS and the IPS Center in 2012. He received awards from the Society of Instrument and Control Engineers (Japan) in 1987 and 1994, and from the Japan Institute of Invention and Innovation in 1985 and 1987. He is a fellow of the Society of Instrument and Control Engineers (Japan). Dr. Ueda is also a member of the Institute of Electrical Engineers (Japan) and the Society of Instrument and Control Engineers (Japan). 